\title{
Gray-Scale Image Compression Techniques: A Review
}

\author{
Asawari Kulkarni \\ Computer Department, \\ P. E. S. Modern College of Engineering, \\ Shivajinagar, Pune-05
}

\author{
Aparna Junnarkar \\ Computer Department, \\ P. E. S. Modern College of Engineering, \\ Shivajinagar, Pune-05
}

\begin{abstract}
Multimedia data are large in size as compared to the plain-text data. And hence to transmit them over a low-bandwidth communication channel, it needs to be compressed. In this paper, focus is on compressing gray-scale images. Here two types of compression techniques are explained: Lossy and Lossless. Within lossy techniques, there are three techniques: Fractal encoding, Discrete Cosine Transform (DCT), and Discrete Wavelet Transform (DWT). And Following are lossless techniques: Arithmatic Encoding, Run Length Encoding (RLE), and Huffman Encoding. The efficiency of compression process is estimated using Compression Ratio (C.R.) and the quality of reconstructed image which is generated after decompression process is calculated using Peak-Signal-to-Noise Ratio (P.S.N.R.).
\end{abstract}

\section{General Terms}

Image Compression, Lossless, Lossy

\section{Keywords}

Gray-scale image, redundancy, compression, decompression lossy, lossless, PSNR, CR, MSE

\section{INTRODUCTION}

A gray-scale image can be seen as a two dimensional matrix of its pixel values. To transmit an image data in an efficient form, it needs to be compressed. If a sender sends a gray-scale image over a low communication channel bandwidth then the image is compressed by channel provider by applying compression algorithm on it. Then compressed image data is given to the intended receiver and receiver decompresses it to get the original gay-scale image.

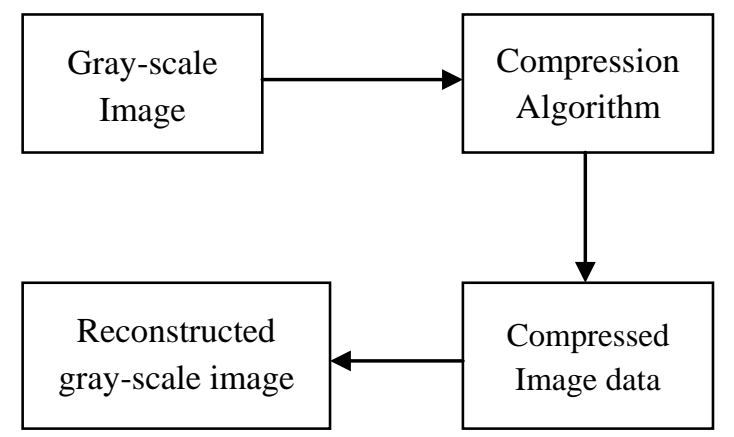

Fig. 1 Gray-scale image compression

There are three types of redundancies in image data: interpixel redundancy, coding redundancy, and psycho-visual redundancy [10]. Inter-pixel redundancy: In neighboring pixels of image, there is a correlation, and they are not independent. It is nothing but an inter-pixel redundancy. It can be explored by predicting pixel values from neighbor pixels. Coding redundancy: In this, the repetitive image data called as codes are removed and for all codes only single code is kept in compressed data. It is explored using Look-Up Tables.
Psycho-visual redundancy: Human eye cannot respond to visual information with equal sensitivity. This is psychovisual redundancy and is exploited in algorithms such as DCT.

Compressing a gray-scale image can benefit in many ways:

1. Cost of transferring large image over lowbandwidth channel is reduced.

2. Transmission efficiency is increased.

3. Space required to store an image is reduced.

4. Compressed image cannot be accessed by unlawful monitors and hence it provides some level of security.

\section{LOSSLESS IMAGE COMPRESSION TECHNIQUES}

In lossless image compression techniques, the quality of the reconstructed image received at receiver after decompression is very good, almost equal to input gray-scale image. Lossless techniques encode the original input gray-scale image to compress it. But with these techniques, it is not possible to achieve a good compression ratio because there is no loss of data at the time of compression procedure. The lossless compression techniques are explained below.

\subsection{Arithmetic Encoding}

Arithmatic technique encodes input gray-scale image information with a string that consists of fractional values between 0 and 1 on the Real number line [7]. Frequently used data within an image is stored with less number of bits and less frequently used image data is stored with more number of bits; hence achieving smaller size of encoded data. This technique is simple in operation and in computations but it is not efficient in compressing the image data.

In a paper [7], Sulthana et al. have considered Arithmatic encoding for Lena image given in fig.1. At the receiver side, they calculated value of PSNR as $30.16 \mathrm{db}$.

\subsection{Run Length Encoding (RLE)}

RLE works on the fact that the same image data can occur in many consecutive image data elements. As a gray-scale image pixel values consists of different intensity values of with only black and white color, it is easy one to use RLE technique [8]. In this technique, input gray-scale image is considered as a two-dimensional matrix of image pixel values and the compression is done with following steps:

1. Values in input image matrix are stored in a vector.

2. Reset four LSBs of each vector by shifting each value to 4 bits to the right and back to the left 
3. The repetitive data in image, called as runs, is replaced with a sequence $\left\{d_{i}, C_{i}\right\}$, where $d_{i}$ is repetitive data and $C_{i}$ is count by which it is repeated.

4. Combine each $d_{i}$ with its frequency i.e. $C_{i}$.

5. At the receiver side, image is decompressed by isolating the 4 MSBs and 4 LSBs.

6. Then $d_{i}$ is replaced with original value and using $C_{i}$ the original image data is retrieved.

The Run Length Encoding performs good and easily for gray scale images but it degrades for image files that don't have recurring sequences.

In the paper [8], Rafeeq et al. implemented RLE on the given image and estimated the value of CR as 0.55 and PSNR value as $29.14 \mathrm{db}$

\subsection{Huffman Encoding}

Huffman encoding technique is invented by David Huffman and is variable length coding. In this, for frequently occurring data in image, the short length code words are used and for less frequent data, longer code words are used [9]. In Huffman encoding, image is compressed with the help of Binary tree. Leaf nodes of the tree are image data and their probabilities are at the side.

Steps in Huffman encoding are explained below,

1. Calculate the probability of each value in input image data.

2. Arrange these probabilities in descending order.

3. Merge the lower probabilities and repeat this until only two probabilities are left.

4. This generated binary tree is the code dictionary.

5. Assign shorter code words to value of image data with high probability and longer code words to image data having less probability. These code words represent compressed image data.

6. Image decompression and reconstruction is done with the help of generated binary tree.

Huffman encoding process is efficient and simple. It gives better reconstructed image after decompression. But Binary tree implementation can be replaced with higher order trees to get improved compression.

In the paper [9], Kumar et al. experimented Huffman encoding for different gray-scale images including given Lena image. For this given image, the obtained PSNR value is $44.611 \mathrm{db}$ and $\mathrm{CR}$ value is 0.138 .

\section{LOSSY IMAGE COMPRESSION TECHNIQUES}

In Lossy image compression techniques, the redundant image data is completely removed. Therefore these techniques are used to achieve higher compression. But there is loss of image data in these techniques and hence they are called as lossy compression techniques. In this, after decompression, the quality of reconstructed image is degraded as compared to original input gray-scale image. There are three lossy compression techniques and those are explained below.

\subsection{Fractal Encoding}

Fractals are repetitive occurring structures in image data. They are similar to each other in terms of size, rotation, or orientation, etc. [2]. This redundant data is removed from the image to be compressed and hence the name fractal encoding.

In fractal encoding, following steps are performed:

1. Divide an input gray-scale image into nonoverlapping blocks of size $4 \times 4$, called as Range blocks $\left(\mathrm{R}_{\mathrm{i}}\right)$.

2. Select a Domain block $\left(D_{i}\right)$ from the image of size twice the Range block.

3. Partition the Domain block $D_{i}$ and calculate its Affine transform. The result is $\mathrm{D}_{\mathrm{i}}{ }^{1}$

4. Calculate the mean square root of $R_{i}$ and $D_{i}{ }^{1}$ to estimate error e between two blocks. If e is less than possible tolerant error then go to step 5, else divide the $\mathrm{D}_{\mathrm{i}}{ }^{1}$ into four equal blocks and repeat the process.

5. Record the information collected till now regarding fractals and stop.

6. To decompress the image, follow above process in the reverse order and get the reconstructed image.

The fractal compression technique has long encoding time. Also, it is computationally expensive because each range block is compared to all domain blocks. In the paper [1], Veenadevi et al. have applied fractal encoding on given image and estimated PSNR value as $11.91 \mathrm{db}$ and CR as 3.2.

\subsection{Discrete Cosine Transform (DCT)}

The image in spatial domain is transformed into frequency domain using DCT. The input image is represented in twodimensional matrix as $\mathrm{P}(\mathrm{i}, \mathrm{j})$. Then the Discrete Cosine transform is applied on this matrix to get $\mathrm{P}^{\prime}(\mathrm{i}, \mathrm{j})$. The DCT is discrete-time version of Fourier transform [4] and is represented with formula given below,

$$
\begin{aligned}
& f(u, v) \\
& =\frac{2}{N} C(u) C(v) \sum_{x=0}^{N-1} \sum_{y=0}^{N-1} \cos \left[\frac{\Pi(2 x+1)}{16}\right] \cos \left[\frac{\Pi(2 y+1)}{16}\right]
\end{aligned}
$$

Here,

Image is divided into $\mathrm{N}$ blocks of size $4 \times 4,8 \times 8,16 \times 16$, or $32 \times 32$

$\mathrm{u}=0,1,2 \ldots \mathrm{N}-1$ and $\mathrm{v}=0,1,2 \ldots \mathrm{N}-1$

This helps to reduce the local spatial correlation between image pixels. Then the quantization is applied on these blocks to remove redundant image data. To get the original image at receiver side, first de-quantization and the inverse DCT is applied on the compressed image data.

Because of the quantization process, the quality of reconstructed image and compression ratio is improved [4]. In the paper [3], Pandey et al. 2D-DCT transformation is applied on given image and calculated PSNR value as $31.95 \mathrm{db}$ and compression ratio as 0.92 .

\subsection{Discrete Wavelet Transform}

Image watermarking is a technique of copyright protection and is done using DWT. Image watermarking consists of decomposing original input gray-scale image into four subbands as LL, LH, HL, and $\mathrm{HH}$ and embedding watermark with one of these sub-bands [6]

In DWT compression technique, input image is processed by 2D-filters in both dimensions of the image. These filters 
divide the image into four sub-bands as LL1, LH1, HL1, and HH1. The LL1 gives the coarser level of information and hence it is again sub-divided into LL2, LH2, HL2, and HH2. This division can be done maximum up to 5 levels.

\begin{tabular}{|c|c|c|c|}
\hline LL3 & HL3 & \multirow{2}{*}{ HL2 } & \multirow{3}{*}{ HL1 } \\
\hline LH3 & HH3 & & \\
\hline \multicolumn{2}{|c|}{ LH2 } & $\mathrm{HH} 2$ & \\
\hline \multicolumn{3}{|c|}{ LH1 } & HH1 \\
\hline
\end{tabular}

Fig. 2 Three level decomposition

Then quantization is applied to get wavelet coefficients and the entropy coding is applied on wavelet coefficients to get the compressed image data [6].

In the paper [5], Navnidhi et al. experimented DWT on the given Image and got PSNR value as $51.46 \mathrm{db}$.

\section{PERFORMANCE PARAMETERS}

The efficiency of compression algorithm is estimated by the amount of image data that is compressed and the quality of reconstructed image after decompression. The compression efficiency can be estimated using Compression ratio and the quality of reconstructed image can be calculated using PSNR.

\subsection{Compression Ratio (CR)}

Less the size of compressed data, more is the compression efficiency. This efficiency is calculated using Compression Ratio. It is the ratio of uncompressed data to compressed data and is measured in percentage. Compression ratio tells how much the image data is compressed. The formula for CR is:

$$
\text { Compression Ratio }=\frac{\text { Uncompressed Data }}{\text { Compressed Data }}
$$

\subsection{Mean-Squared Error (MSE)}

The MSE is the cumulative squared error between the compressed and the original image. A lower value of MSE means lesser error and good quality of reconstructed image. For a noise-free image $I$ of size $m \times n$ and its noise approximation $\mathrm{K}, \mathrm{MSE}$ is defined as:

$$
M S E=\frac{1}{m n} \sum_{i=0}^{m-1} \sum_{j=0}^{n-1}[I(i, j)-K(i, j)]^{2}
$$

\subsection{Peak-Signal to Noise Ratio (PSNR)}

It is the ratio between the maximum possible power of original image and the power of noise introduced because of compression. Many signals have wide range hence PSNR is expressed in terms of the decibel scale $(\mathrm{db})$. Higher the PSNR value better is the quality of reconstructed image. PSNR is defined using the Mean Squared Error (MSE). The PSNR of reconstructed image is calculated using following formula:

$$
P S N R=10 \log _{10}\left(\frac{M A X_{I}^{2}}{M S E}\right)
$$

Here,

MAX is the maximum possible pixel value of the image.

Using these parameters, performance of compression algorithm can be estimated and hence these parameters are essential to consider while compressing the image data.

\section{CONCLUSION}

After the study of gray-scale image compression techniques, it has been observed that among lossless compression techniques, Huffman Encoding gives better quality of reconstructed image and among lossy compression techniques; Discrete Wavelet Transform performs efficiently in producing compressed data.

In the future, a hybrid technique of lossless and lossy image compression techniques can be used to achieve better reconstructed image and efficient compression.

\section{ACKNOWLEDGMENTS}

Every orientation work has an imprint of many people and it becomes the duty of author to express deep gratitude for the same. I take this opportunity to express my deep sense of gratitude towards my esteemed guide Prof. (Ms) Aparna Junnarkar for giving me this splendid opportunity to present this paper.

\section{REFERENCES}

[1] Veenadevi. S. V., A. G. Ananth, "Fractal Image Compression of Satellite Imageries," International Journal of Computer Applications, September 2011.

[2] Hitashi Gaganpreet Kaur Sugandha Sharma, "Fractal image compression- a review," International Journal of Advanced Research in Computer Science and Software Engineering, February 2014.

[3] Xinpeng Zhang, Yanli Ren, Liquan Shen, Zhenxing Qian, and Guorui Feng, "Compressing Encrypted Images With Auxiliary Information," IEEE Transactions on Multimedia, August 2014

[4] Dr. S. S. Pandey, Manu Pratap Singh, Vikas Pandey, "Block Wise Image Compression \& Reduced Blocks Artifacts Using Discrete Cosine Transform ,' International Journal of Scientific and Research Publications, March 2015.

[5] Tim Bruylants, AdrianMunteanu, PeterSchelkens, "Wavelet based volumetric medical image compression," Elsevier, December 2014.

[6] Navnidhi Chaturvedi, Dr. S. J. Basha, "Comparison of Digital Image watermarking Methods DWT \& DWTDCT on the Basis of PSNR," International Journal of Innovative Research in Science, Engineering and Technology, December 2012.

[7] S. Nigar Sulthana, Mahesh Chandra, "Image Compression with Adaptive Arithmetic Coding," International Journal of Computer Applications, 2011.

[8] Rafeeq AL-HASHEMI, Ayman AL-DMOUR, Fares FRAIJ, Ahmed MUSA, "A Grayscale Semi-Lossless Image Compression Technique Using RLE,” Journal of Applied Computer Science \& Mathematics, 2011.

[9] Sharan Kumar, Dr.Jayadevappa, Santosh .D.Bhopale, Radhika R. Naik, "Implementation of Huffman Image Compression And Decompression Algorithm," International Journal Of Innovative Research In Electrical, Electronics, Instrumentation And Control Engineering, April 2014. 
International Journal of Computer Applications (0975 - 8887)

Volume 131-No.13, December2015

[10] Gaurav Vijayvargiya, Dr. Sanjay Silakari, Dr.Rajeev Pandey, "A Survey: Various Techniques of Image Compression," International Journal of Computer Science and Information Security, October 2013.

[11] Anshuma Patel, Sanjiv Kumar Shriwastava, "Parameterized Comparative Analysis of Various Lossy and Lossless Image Compression Practices,"
International Journal of Advanced Research in Computer and Communication Engineering, February 2015.

[12] Rahul Shukla, Narender Kumar Gupta, "Image Compression through DCT and Huffman Coding Technique," International Journal of Current Engineering and Technology, May 2015. 